\title{
Factores Psicosociales Relacionados con la Calidad de Vida en Salud en Pacientes Hemodializados
}

\author{
Psychosocial Factors Linked to Health Related Quality of Life in Hemodialysis Patients
}

\author{
Alfonso Urzúa \\ Universidad Católica del Norte, Chile \\ Ruth Pavlov \\ Centro de Diálisis NORDIAL, Antofagasta, Chile \\ Roberto Cortés \\ y \\ Vanessa Pino \\ Universidad Católica del Norte, Chile
}

(Rec: 20 de abril de 2010; Acept: 18 de marzo de 2011)

\begin{abstract}
Resumen
Objetivo: analizar la relación existente entre las variables edad, tiempo en tratamiento, sexo, apoyo familiar percibido, salud mental, estado de salud percibido y la calidad de vida (CV) Método: Se evaluaron 128 pacientes del Hospital Clínico Regional y de un Centro de diálisis privado, ambos de la ciudad de Antofagasta. Se utilizó el cuestionario específico para CV en enfermedad renal KDQOL-36 TM y los instrumentos Apgar Familiar y GHQ-28. Se analiza la relación existente entre cada dimensión de la CV evaluada y los factores mencionados. Resultados: No se encuentra relación entre la CV el tiempo viviendo con la enfermedad, como tampoco con la edad o el sexo. Las diversas dimensiones en salud mental son de las evaluadas las que presentan un mayor grado de correlación con las dimensiones de calidad de vida. Conclusiones: La salud mental es un elemento modulador de la CV, en tanto otros como el sexo, el tiempo de diálisis o la edad de los participantes no constituyeron elementos relevantes en esta evaluación.

Palabras clave: Insuficiencia renal crónica terminal, hemodiálisis, calidad de vida relacionada con la
\end{abstract} salud, KDQOL-36 TM.

\begin{abstract}
Objective: To analyze the relationship between the variables age, time in treatment, gender, perceived family support, mental health, perceived health status and quality of life (QoL). Method: We studied 128 patients in Public Hospital and a private dialysis center, both in the city of Antofagasta. We used the questionnaire for kidney disease $36^{\mathrm{TM}}$ KDQOL-36 TM , the Apgar Family and GHQ-28. We evaluated the relationship between each dimension of the QoL and the factors mentioned. Outcomes: No relationship was found between the QoL and time living with the disease, gender and age. The several dimensions of mental health is assessed to have a greater correlation with the dimensions of quality of life. Conclusions: Mental health is a modulator of the QoL, while others such as gender, length of dialysis and the age of the participants were not factors relevant to this assessment.
\end{abstract}

Key words: Chronic renal failure, hemodialysis, health related quality of life, KDQOL-36 TM. 


\section{Introducción}

La insuficiencia renal crónica terminal (IRCT) es una enfermedad que implica la pérdida gradual de la función de los riñones, pudiendo llegar a ser terminal (Contreras, Esguerra, Espinoza, Gutiérrez \& Fajardo, 2006). De los tratamientos utilizados la hemodiálisis (HD) se emplea en el 90\% de los pacientes.

En la última década se ha apreciado un crecimiento considerable de pacientes con IRCT y por ende de personas sometidas a HD. En Chile, en 1998 hubo 5.594 pacientes, mientras que en el año 2008 se registraron 13.636 pacientes (Poblete, 2008).

La HD genera cambios en los estilos de vida y en las conductas cotidianas de las personas que se someten a ella, tales como la dieta alimentaria, la ingesta de líquidos y uso de medicamentos (Álvarez, Fernández, Vázquez, Mon, Sánchez \& Rebollo, 2001). El tratamiento produce también efectos adversos a nivel físico (Contreras, Esguerra, Espinoza \& Gómez, 2007) y psicológicos, tales como depresión, ansiedad y trastornos de conducta (Álvarez et al., 2001). En pacientes con tratamiento sustitutivo renal además se observan efectos a nivel psicosocial, tales como problemas maritales, disfunción sexual y problemas socioeconómicos (Daugirdas, 2007). Todos estos cambios y efectos inciden directamente en la Calidad de Vida (CV) de las personas.

La Calidad de Vida relacionada con la Salud (CVRS) puede ser definida como el nivel de bienestar derivado de la evaluación que la persona realiza de diversos dominios de su vida, considerando el impacto que en éstos tiene su estado de salud (Urzúa, 2010). Esta incluye la evaluación que realiza cada individuo respecto a la salud y al grado de funcionamiento para realizar las actividades cotidianas (Schwartzmann, 2003).

La CVRS en pacientes dializados ha sido estudiada por diversos investigadores, reportándose en la literatura diversos elementos que influyen en ésta. El mayor porcentaje de estas investigaciones se ha realizado en España y Estados Unidos, siendo escasas en países latinoamericanos. En Chile, la IRCT se ha estudiado desde el punto de vista epidemiológico, de evolución clínica, complicaciones a largo plazo, morbilidad, mortalidad, entre otras (Poblete, 2008), encontrándose solo un estudio que evalúa la CVRS en la Región del Bío-Bío (Zúñiga, Dapueto, Müller, Kirsten, Alid \& Ortiz, 2009), en la cual se evalúa solo la relación existente entre la CV y variables biomédicas y sociodemográficas.

En este marco, el objetivo de esta investigación fue analizar la relación existente entre variables psicosociales que la literatura menciona con mayor frecuencia vinculadas a la CVRS en HD: edad, tiempo en tratamiento, sexo, apoyo familiar percibido, salud mental, estado de salud percibido y la CVRS en pacientes hemodializados en población chilena, a fin de pesquisar qué factores aparecen como influyentes de ésta.

Los resultados del estudio de estas variables podrían repercutir favorablemente en el bienestar del paciente renal, toda vez que al conocer aquellas dimensiones modificables vinculadas a la CVRS, la intervención en dichas áreas podría ser utilizada como recurso para una estrategia terapéutica integral, destinada a mejorar la CVRS. Se debe considerar además la importancia de implementar programas de ayuda psicológica para los pacientes, las redes de apoyo, además de capacitaciones para el equipo sanitario implicados en el tratamiento sustitutivo renal, y de esta forma lograr que conozcan las herramientas necesarias para evitar el deterioro y mejorar la CVRS.

Dada la literatura revisada, se espera encontrar que: (a) los hombres presenten una mejor CVRS que las mujeres (Álvarez et al., 2001; Ruiz, Roman, Martin, Alferez \& Prieto, 2003; Vázquez et al., 2004; Wehbe, Salazar, Vaccaro, Wehbe \& Guerrero, 2004); (b) conforme avance la edad de los pacientes hemodializados, la CVRS disminuya (Lopes et al., 2007; Moreno et al., 2004; Patel, Peterson \& Kimmel, 2005; Ruiz et al., 2003; Wehbe et al., 2004); (c) Los pacientes con mayor tiempo en tratamiento de hemodiálisis presenten una peor CVRS (Muñoz, Oto, Barrio \& Fernández, 2006); (d) los pacientes que perciben tener una familia normofuncional, presentan una mejor CVRS que los pacientes que perciben una familia disfuncional (Rodríguez \& Rodríguez, 2004; Wehbe et al., 2004); (e) la presencia de alteraciones emocionales se relaciona inversamente con la CVRS (Álvarez et al., 2004; Atencio, Nucette, Colina, Sumalave, Gómez \& Hinestroza, 2004; Morales, Salazar, Flores, Bochicchio \& López, 2008; Moreno et al., 2004; Muñoz et al., 2006; Ortega \& Martínez, 2002).

\section{Método}

La presente investigación se enmarca bajo un tipo de diseño no experimental de tipo transeccional correlacional.

\section{Participantes}

Participaron en el estudio el 95\% del total de pacientes de un Centro de diálisis privado y el $76 \%$ del total de pacientes que acudían a recibir HD en el Hospital Clínico Regional de la ciudad de Antofagasta. De los 128 pacientes encuestados, el 50,8\% fueron hombres y el 49,2\% mujeres. La edad osciló entre 18 a 81 años (media=56 años; $\mathrm{DE}=13.80$ años). El tiempo promedio de tratamiento en HD fue de 49 meses (DE=46 meses; mínimo 1 mes, máximo 205 meses). Se consideró como criterio de inclusión, además del diagnóstico clínico de IRCT, el ser mayor de edad y estar en condiciones físicas y psicológicas adecuadas para responder los cuestionarios. 


\section{Procedimiento}

Luego de obtener los permisos en las instituciones para realizar la investigación y la aprobación del Comité de ética de la Universidad Católica del Norte, se seleccionaron los participantes que cumpliesen con los criterios de inclusión. Estos fueron informados acerca del objetivo de la investigación, firmando su consentimiento para participar en el estudio.

Los instrumentos fueron aplicados individualmente mientras duraba el proceso de HD. El tiempo promedio de duración fue de 45 minutos. Una vez recolectados los datos, estos fueron ingresados a una base de datos construida en SPSS versión 14.0 para los análisis posteriores. Se obtuvieron datos de fiabilidad de los instrumentos utilizados a través del alfa de Cronbach. Posteriormente se realizaron análisis descriptivos y de frecuencia, así como también, correlaciones bivariadas entre las dimensiones del KDQOL$36^{\mathrm{TM}}$ y el resto de los instrumentos utilizados en el estudio. Se utilizaron pruebas $\mathrm{T}$ para evaluar la diferencia de medias dadas por el sexo, Institución y las categorías aportadas por el Apgar familiar.

\section{Instrumentos}

\section{KDQOL-36 TM}

El Kidney Disease Quality of Life es un cuestionario específico para pacientes con enfermedad renal en diálisis (Hays, Kallich, Mapes, Coons \& Carter, 1994). Existe una versión abreviada- Kidney Disease Quality of Life Short Form- elaborada y validada por la Universidad de Arizona (Hays et al., 1995), a partir del KDQOL y el MOS SF-36 (Medical Outcomes Study Short Form General Health Survey). La versión adaptada al español fue desarrollada el año 1997 (Hays, Amin, Alonso et al, 1997) y validada preliminarmente el año 1998 (García, López, De Álvaro, Alvárez, Alonso, 1998).

El KDQOL-36 TM, es la versión paralela del KDQOL$\mathrm{SF}^{\mathrm{TM}}$. Este cuestionario fue traducido y validado en España por Jordi Alonso et al. (2008).
Esta versión la integran 36 preguntas y está constituida por un módulo genérico que incluye el SF-12 y tres módulos específicos sobre la enfermedad al riñón: síntomas (12 reactivos), efectos de la enfermedad renal (8 reactivos) y peso (carga) de la enfermedad renal (4 reactivos).

Las opciones de respuesta forman, en su mayoría, escalas de tipo Likert que evalúan intensidad o frecuencia con rangos de respuesta entre tres y cinco, dependiendo del ítem. Cada pregunta está codificada numéricamente y su puntaje luego es trasformado en una escala de 0 a 100, en donde un mayor valor refleja una mejor CVRS (Morales et al., 2008). $\mathrm{El}$ análisis de fiabilidad del instrumento arrojó en esta investigación un $\alpha$ de Cronbach $=.87$ para la escala total.

\section{Apgar Familiar}

Fue diseñado por Smilkstein (1978), para medir la funcionalidad familiar. La versión española de Apgar Familiar, está compuesta por 5 preguntas que permiten obtener un puntaje total que posibilita la clasificación en dos niveles de funcionamiento: disfuncionalidad y normofuncionalidad. La consistencia interna del instrumento en esta investigación a través del $\alpha$ de Cronbach fue de .80 .

\section{GHQ-28}

Se utilizó la versión de 28 reactivos del Goldberg Health Questionnaire (GHQ-28), el cual evalúa el estado mental, detectando trastornos de al menos 2 semanas de duración (Goldberg, 1979; Lobo, Pérez y Artal, 1986). Las preguntas se agrupan en 4 dimensiones: síntomas somáticos, ansiedad e insomnio, disfunción social y depresión grave. El análisis de fiabilidad del instrumento arrojó en la presente investigación un $\alpha=.78$ para la escala total.

\section{Resultados}

\section{Participantes}

De los 128 pacientes con diagnóstico de IRCT, 87 (68\%) fueron encuestados en un Centro de Diálisis privado, en tanto 41 desde el Hospital clínico Regional. En la Tabla 1, se muestran las variables sociodemográficas estudiadas.

Tabla 1

Descripción de la muestra.

\begin{tabular}{lrrrrrr}
\hline & \multicolumn{1}{c}{ Centro Diálisis Privado } & Hospital Clínico Regional & & Total \\
\hline Hombres & 47 & $54 \%$ & 16 & $39 \%$ & 63 & $49,2 \%$ \\
Mujeres & 40 & $46 \%$ & 25 & $61 \%$ & 65 & $50,8 \%$ \\
Media Edad (años) & 58.0 & $\mathrm{DE}=13.90$ & 51.9 & $\mathrm{DE}=12.75$ & 56.10 & $\mathrm{DE}=13.80$ \\
Media meses en Hemodiálisis & 44.5 & $\mathrm{DE}=39.42$ & 60.1 & $\mathrm{DE}=57.69$ & 49.43 & $\mathrm{DE}=46.30$ \\
\hline
\end{tabular}




\section{Calidad de vida relacionada con la Salud}

En la Tabla 2 se pueden observar las medias reportadas para las distintas dimensiones evaluadas por el KDQOL$36^{\mathrm{TM}}$. No se observan diferencias estadísticamente significativas entre las medias de los hombres y mujeres, con excepción de la dimensión síntomas, donde la media de las mujeres es menor que la de los hombres $(\mathrm{t}(126)=2,809$; $\mathrm{p}<.05)$.
Al analizar la relación existente con la edad, la única dimensión que correlaciona significativamente y de manera inversa con ésta, es la dimensión física del SF-12 ( $\mathrm{r}=-0,279$; $\mathrm{p}<.05)$.

No se encuentra relación entre los puntajes de las diversas dimensiones y el tiempo en HD.

Tabla 2.

Medias por dimensiones para sexo y tipo de establecimiento.

\begin{tabular}{lccccc}
\hline & $\begin{array}{c}\text { Hombres } \\
(\mathrm{n}=63)\end{array}$ & $\begin{array}{c}\text { Mujeres } \\
(\mathrm{n}=65)\end{array}$ & $\begin{array}{c}\text { Hospital } \\
(\mathrm{n}=41)\end{array}$ & $\begin{array}{c}\text { Centro Privado } \\
(\mathrm{n}=87)\end{array}$ & $\begin{array}{c}\text { Total } \\
(\mathrm{n}=128)\end{array}$ \\
\hline Síntomas & $77.11+14.60$ & $69.16+17.25$ & $70.62+19.12$ & $74.23+14.98$ & $73.07+16.43$ \\
Efectos enfermedad & $59.17+23.35$ & $56.54+23.57$ & $58.31+25.20$ & $57.61+22.67$ & $57.83+23.41$ \\
Peso enfermedad & $43.94+29.20$. & $42.30+30.09$ & $42.53+34.29$ & $43.39+27.24$ & $43.11+29.55$ \\
SF12 Físico & $38.71+10.63$ & $35.25+9.41$ & $38.33+9.81$ & $36.31+10.28$ & $36.95+10.14$ \\
SF12 Mental & $48.87+11.17$ & $45.18+11.88$ & $45.59+11.65$ & $47.65+11.64$ & $46.99+11.64$ \\
\hline
\end{tabular}

Calidad de vida relacionada con la Saludy Funcionamiento Familiar

La Tabla 3 contiene las medias obtenidas en cada una de las dimensiones según la clasificación generada a partir del Apgar familiar. Pese a que la media en todas las dimensiones es superior en el grupo de familias categorizadas como normofuncionales, esta solo es significativamente mayor en este grupo comparada con los categorizados como disfuncionales en las dimensiones sintomatología ( $\mathrm{t}=-2$ ,717(126); $<<.05)$, carga de enfermedad $(\mathrm{t}=-2,261(126)$; $\mathrm{p}<.05)$ y salud mental $(\mathrm{t}=-2,336(126) ; \mathrm{p}<.05)$.

Tabla 3 .

Medias en dimensiones por tipo de funcionamiento familiar.

\begin{tabular}{lcc}
\hline & $\begin{array}{c}\text { Familia disfuncional } \\
(\mathrm{n}=16)\end{array}$ & $\begin{array}{c}\text { Familia Normofuncional } \\
(\mathrm{n}=112)\end{array}$ \\
\hline Síntomas & $62.88 \pm 15.52$ & $74.53 \pm 16.10$ \\
Efectos enfermedad & $50.78 \pm 18.10$ & $58.84 \pm 23.98$ \\
Carga enfermedad & $27,73 \pm 26.12$ & $45.31 \pm 29.46$ \\
SF12 Físico & $34.70 \pm 7.30$ & $37.27 \pm 10.47$ \\
SF12 Mental & $40.75 \pm 10.78$ & $47.89 \pm 11.53$ \\
\hline
\end{tabular}

\section{Calidad de vida relacionada con la Salud y Salud Mental}

Al analizar la relación existente entre las dimensiones de CVRS y las dimensiones de salud mental generadas a partir del GHQ-28 (Tabla 4), se observa que todas éstas se correlacionan negativa y significativamente con la depresión y la disfunción social. No se encuentra relación entre la ansiedad y el dominio de salud física, ni entre los síntomas somáticos y la salud física y mental reportada por el GHQ -28. 
Tabla 4.

Correlación dimensiones CVRS y dimensiones $G H Q-28(n=128)$.

\begin{tabular}{lccccc}
\hline & & Síntomas somáticos & Ansiedad e Insomnio & Disfunción Social & Depresión Grave \\
\hline Síntomas & $\mathrm{r}$ & $-.329(* *)$ & $-.232(* *)$ & $-.211(*)$ & $-.336(* *)$ \\
Efectos Enfermedad & $\mathrm{r}$ & $-.302(* *)$ & $-.286(* *)$ & $-.351(* *)$ & $-.336(* *)$ \\
Peso enfermedad & $\mathrm{r}$ & $-.197(*)$ & $-.362(* *)$ & $-.321(* *)$ & $-.397(* *)$ \\
SF12 Físico & $\mathrm{r}$ & -.126 & -.111 & $-.229(* *)$ & $-.260(* *)$ \\
SF12 Mental & $\mathrm{r}$ & -.154 & $-.248(* *)$ & $-.354(* *)$ & $-.342(* *)$ \\
\hline
\end{tabular}

$\mathrm{r}=$ correlación de Pearson; $* *$ La correlación es significativa al nivel .01 (bilateral). $*$ La correlación es significativa al nivel 0.05 (bilateral).

\section{Discusión}

Se analizaron diversos factores que la literatura mencionaba vinculados a la calidad de vida en pacientes en hemodiálisis. Aun cuando investigaciones previas señalan que los hombres presentan una mejor CVRS que las mujeres (Álvarez et al., 2001; Ruiz et al., 2003; Vázquez et al., 2004; Wehbe et al., 2004), los datos encontrados en esta investigación no aportan mayor evidencia a esta idea, ya que aunque las mujeres obtuvieron puntuaciones más bajas que los hombres en todas las dimensiones, estas diferencias no son significativas, con excepción de la dimensión síntomas somáticos. El hecho de que las mujeres perciban mayor molestia en comparación a los hombres ha sido reportado en otras investigaciones (Contreras et al., 2006; Vázquez et al., 2004). Aun cuando la mayoría de las investigaciones en CVRS en enfermedades crónicas no reportan diferencias entre la percepción de hombres y mujeres (Urzúa, 2008), algunos autores han sugerido que las mediciones no necesariamente reflejan las experiencias objetivas y subjetivas de la mujer en salud, ya que esta última no es neutral al género, siendo necesaria la construcción de instrumentos con este enfoque (Eckermann, 2000). Otra posible hipótesis tiene que ver con que las mujeres se permiten expresar con mayor libertad que los hombres las molestias ocasionadas por la sintomatología, lo cual no quiere decir que los hombres no sientan la misma incomodidad, sino que no la expresan. Esto abre futuras líneas de investigación en torno a la expresión y vivencias de la enfermedad condicionadas por el rol de género para esta enfermedad en particular.

Se esperaba que a medida que los participantes tuviesen mayor edad autorreportasen una menor CVRS (Lopes et al., 2007; Moreno et al., 2004; Patel et al., 2005; Ruiz et al., 2003; Wehbe et al., 2004), sin embargo, esta solo se encontró relacionada con la dimensión de salud física del SF-12 y no con las dimensiones específicas del KDQOL. El hecho de que la edad no se constituya como un factor determinante de la CVRS, ha sido reportado por otros investigadores (Contreras et al., 2006), ya que esta solo se encuentra relacionada con la capacidad física, la cual se encuentra más deteriorada conforme a mayor edad de la persona, tenga o no una enfermedad crónica.

Se esperaba que los participantes con mayor tiempo en tratamiento de hemodiálisis presentaran una peor CVRS, dado el desgaste propio de la enfermedad (Muñoz et al., 2006), sin embargo, en los participantes en este estudio el tiempo en tratamiento no interviene de manera significativa en la evaluación de su CVRS. Este hecho puede deberse al proceso de adaptación a la enfermedad, fenómeno que ha sido observado en otras patologías crónicas (Urzúa, 2008). Sobre este punto se abre otra línea de investigación, toda vez que se hace necesaria la realización de estudios longitudinales que permitan evaluar esta adaptación y el impacto de la enfermedad a través del tiempo, a fin de determinar si ambas son variables que interviene la CVRS de manera conjunta.

La gran mayoría de los pacientes declara tener una familia normofuncional. Pese a que se hipotetizaba que los pacientes que perciben tener una familia normofuncional, presentarían una mejor CVRS que los pacientes que perciben una familia disfuncional (Rodríguez \& Rodríguez, 2004; Wehbe et al., 2004), esta hipótesis se verificó a medias, sin resultados concluyentes. El tipo de estructura familiar solo fue significativo en su relación con la presencia de síntomas, la carga de la enfermedad y la salud mental, actuando la normofuncionalidad percibida de la familia como un agente protector que afecta la CVRS del paciente renal, especialmente en la percepción de síntomas, el peso de la enfermedad y la percepción de sintomatología asociada a problemas de salud mental. Estos datos coinciden con estudios previos relacionados a la función familiar de los pacientes (Rodríguez, Castro \& Merino, 2005; Wehbe et al., 2004).

Con relación a la salud mental de los pacientes, se esperaba encontrar que la presencia de alteraciones emocionales se relacionaría inversamente con la CVRS (Álvarez et al., 2004; Atencio et al., 2004; Morales et al., 2008; Moreno et 
al., 2004; Muñoz et al., 2006; Ortega \& Martínez, 2002). Esta hipótesis pudo ser comprobada, dado que la ansiedad, la disfunción social y la depresión, afectan la CVRS. Donde más comprometida se encuentre la salud física y mental, mayor será la presencia de disfunción social, ansiedad y depresión. Los datos de esta investigación coinciden con trabajos previos (Álvarez et al., 2001; Atencio et al., 2004; Morales et al., 2008; Moreno et al., 2004; Patel et al., 2005) que además refieren que los pacientes con mayores niveles de ansiedad y de depresión tienden a quejarse más de los síntomas, y por consiguiente percibir una baja CVRS.

Los datos obtenidos nos permiten identificar líneas de acción a ser incorporadas en el quehacer del psicólogo de la salud y del psicólogo clínico que trabajan con pacientes dializados, a fin de que a través de la intervención en estas, pueda aumentarse el bienestar de los pacientes que deben someterse al tratamiento de diálisis.

Cabe destacar que al momento de encuestar a los pacientes surgieron nuevas dimensiones que el instrumento no evaluó. Así, en futuras investigaciones se propone abordar la temática de sexualidad en la dinámica de pareja, puesto que la literatura internacional reporta que las disfunciones sexuales son frecuentes en los portadores de IRCT, y éstas podrían influir en la CVRS. Es necesario además incluir dimensiones que permitan evaluar aspectos relacionados con la enfermedad y su impacto en el núcleo familiar.

\section{Referencias}

Alonso J. et al. (2008). Kidney disease quality of life - SF36 ${ }^{T M-}$ Spanish version Cuestionario no publicado, autorización para su utilización solicitada el 06 marzo del 2010 en página del Kidney disease quality of life working group.: http: //www.gim.med.ucla.edu/kdqol/index.htm.

Álvarez, F., Fernández, M., Vázquez, A., Mon, C., Sánchez, R., \& Rebollo, P. (2001). Síntomas físicos y trastornos emocionales en pacientes en programa de hemodiálisis periódicas. Nefrología, 21, 191-199.

Atencio, B., Nucette, E., Colina, J., Sumalave, H., Gómez, F., \& Hinestroza, D. (2004). Evaluación de la depresión y ansiedad en pacientes con insuficiencia renal crónica sometidos a hemodiálisis. Revista Archivos Venezolanos de Psiquiatría y Neurología, 50, 35-40.

Contreras, F., Esguerra, G., Espinosa, J., \& Gómez, V. (2007). Estilos de afrontamiento y calidad de vida en pacientes con insuficiencia renal crónica (IRC) en tratamiento de hemodiálisis. Acta Colombiana de Psicología, 10, 169 -179.

Contreras, F., Esguerra, G., Espinoza, J., Gutierrez, C., \& Fajardo, L. (2006). Calidad de vida y adhesión al tratamiento en pacientes con insuficiencia renal crónica en tratamiento de hemodiálisis. Universitas Psychologica, 5, 487-499.

Daugirdas, J. (2007). Manual de diálisis. Philadelphia: Lippincott Williams \& Wilkins

Eckermann, L. (2000). Gendering indicators of health and well being: Is quality of life gender neutral? Social Indicators Research, 52, 29 - 54.

García, F., López, K., De Álvaro, F., Alvárez, U., Alonso, J., (1998) Salud percibida en pacientes que comienzan tratamiento renal sustitutivo: Validación preliminar de la versión española del KDQOL-SF. Nefrología, 18,66 .

Goldberg, D., Hillier, V. (1979). A scaled version of the General Health Questionnaire. Psychological Medicine, 9, 139-145.
Hays, R., Kallich, J., Mapes, D., Coons, S., Carter, W. (1994). Development of the kidney disease quality of life (KDQOLTM) instrument. Qual Life Res, 3, 329-338.

Hays RD, et al., (1995). Kidney disease quality of life Short form (KDQOLSFTM). Santa Mónica, CA: Rand.

Hays, R. D., Amin, N., Alonso, J., Kallich, J., Coons, S. J., Carter, W. B., Mapes, D. L., \& Kamberg, C. J. (1997). Kidney disease quality of life short form (KDQOL-SF), version 1.2: A manual for use and scoring (Spanish Questionnaire, Spain). P-7928/1. Santa Monica, CA: RAND.

Lobo, A., Pérez-Echeverría, M., Artal, J. (1986). Validity of the scaled version of the General Health Questionnaire (GHQ-28) in a Spanish population. Psychological Medicine, 16, 135-140.

Lopes, A., Bragg, J., Goodkin, D., Fukuhara, S., Mapes, D., Young, E., Gillespie, B., Akizawa, T., Greenwood, R., Andreucci, V., Akiba, T., Held, P., \& Port, F. (2007). Factors associated with health-related quality of life among hemodialysis patients in the DOPPS. Quality of Life Research, 16, 545-557.

Morales, R., Salazar, E., Flores, F., Bochicchio, T., \& López, A. (2008). Calidad de vida relacionada con la salud en los pacientes con tratamiento sustitutivo renal: El papel de la depresión. Gaceta Médica de México, 144, 91-98.

Moreno, E., Arenas, M., Porta, E., Escalant, L., Canto, N., Castell, G., Serrano, F., Sanper, J., Millán, D., \& Cases, J. (2004). Estudio de la prevalencia de trastornos ansiosos y depresivos en pacientes en hemodiálisis. Revista Sociedad Española de Enfermería Nefrológica, 7, 225- 233

Muñoz, R., Oto, A., Barrio, R., \& Fernández, M. (2006). Evolución de la calidad de vida en pacientes en hemodiálisis: Estudio prospectivo a un año. Revista Sociedad Española de Enfermería Nefrológica, 9, 55-58.

Ortega, N., \& Martínez, M. (2002). Bienestar psicológico como factor de dependencia en hemodiálisis. Revista de Enfermería del IMS, 10, 17-20.

Patel, S., Peterson, R., \& Kimmel, P. (2005). The impact of social support on end- stage renal disease. Seminars in Dialysis, 18, 98-102.

Poblete, B. (2008). Hemodiálisis crónica en Chile. XXVIII. Cuenta de Hemodiálisis Crónica en Chile. Sociedad chilena de Nefrología, 1-18.

Rodríguez, M., Castro, M., \& Merino, J. (2005). Calidad de vida en pacientes renales hemodializados. Ciencia y Enfermería, 11, 47-57.

Rodríguez, G., \& Rodríguez, I. (2004). Disfunción familiar en pacientes con insuficiencia renal crónica. Revista Médica del IMS, 42, 97- 102.

Ruiz, M., Román, M., Martin, G., Alférez, M., \& Prieto, D. (2003). Calidad de vida relacionada con la salud en las diferentes terapias sustitutivas de la insuficiencia renal crónica. Revista Sociedad Española de Enfermería Nefrológica, 6, 6-16.

Schwartzmann, L. (2003). Calidad de vida relacionada con la salud: Aspectos conceptuales. Ciencia y enfermería, 9, 9-21.

Smilkstein, G. (198). The family APGAR: A proposal for a family function test and its use by physicians. Journal of family practice, 15, 303-311.

Urzúa, A. (2010) Calidad de Vida relacionada con la Salud: Elementos conceptuales. Revista Medica de Chile, 138, 131 - 138.

Urzúa, A. (2008). Calidad de vida y factores biopsicosociales en patologías médicas crónicas. Terapia Psicológica, 26, 207 - 214.

Vásquez, I., Valderrabano, F., Fort, J., Jofré, R., López, J., Moreno, F., \& Sanz, D. (2004). Diferencias en la calidad de vida relacionada con la salud entre hombres y mujeres en tratamiento en hemodiálisis. Nefrología, 24,167-178.

Wehbe, J., Salazar, J., Vaccaro, Y., Wehbe, I., \& Guerrero, R. (2004). Diálisis y calidad de vida. Archivos venezolanos de psiquiatría y neurología, 50, 27-32.

Zúñiga, C., Dapueto, J., Müller, H., Kirsten, L., Alid, R., \& Ortiz, L. (2009). Evaluación de la calidad de vida en pacientes en hemodiálisis crónica mediante el cuestionario "Kidney Disease Quality of Life (KDQOL36)". Revista Medica Chile, 137, 200 - 207. 